\title{
A MULTIGRID METHOD FOR ELASTO-HYDRODYNAMIC CONTACT SIMULATIONS OF RADIAL SLIDER BEARINGS*
}

\author{
MICHAEL REICHELT ${ }^{\dagger}$, MARKUS WINDISCH $^{\dagger}$, GÜNTER OFFNER ${ }^{\dagger}$, AND SARAH SANTNER ${ }^{\ddagger}$
}

\begin{abstract}
The well-known Reynolds equation is typically used to compute the pressure distribution for elastohydrodynamic contacts of parts, as, for instance, in radial slider bearings. In order to resolve local pressure phenomena like edge loading, a higher spatial resolution is needed. This causes problems for stationary solvers, like Gauss-Seidel iteration, which are well suited for the occurring nonlinearities. These problems can be overcome by applying multigrid methods. Since the Reynolds equation is nonlinear, expensive nonlinear multigrid methods are expected to be required. This paper introduces an approach to combine a linear multigrid method with a Gauss-Seidel solver on the finest level, which yields a similar convergence behavior as a nonlinear multigrid method but at much lower computational cost. The formulations are general so that analogous applications of the Reynolds equation, as, for instance, for axial slider bearings or hydrodynamic piston-liner contacts, are straightforward.
\end{abstract}

Key words. radial slider bearings, Reynolds equation, multigrid

AMS subject classifications. 76A20, 65N55, 76M12

1. Introduction. Lubricated slider bearings of different types are an essential part of various mechanisms. Applications can be found, for instance, in power plants, pumps, transmissions, internal combustion engines, and turbo chargers. Probably the most common type is the radial slider bearing, which is used as a model problem in this paper. The transient simulation of such a bearing is an important task during the development process of the mentioned mechanisms, targeting friction power loss, wear, acoustic excitation, as well as the overall lubrication system. Nonlinear structural properties of the contact parts of journal and shell in combination with nonlinear fluid properties define the characteristic of a bearing. Consequently, applied mathematical models have to consider the dynamics of the overall flexible body system in addition to the detailed properties of the contacts and the lubricant itself. For a general introduction, see [7].

The governing equation for the simulation of the hydrodynamic contact pressure distribution is the Reynolds equation [2]. It yields the oil film pressure distributions over time. An example of a computed 2D pressure distribution at a certain point of time is depicted in Figure 1.1.

Typically, multiple radial slider bearings - and therefore multiple Reynolds equationshave to be considered within a mechanism. Figure 1.2 illustrates a 3D physical model of a $1.9 \mathrm{~L}$ four-cylinder diesel inline engine with a bore of $80 \mathrm{~mm}$ and a stroke of $92.8 \mathrm{~mm}$, as in [7]. In this model 15 bodies and 27 hydrodynamic contacts are used, which leads in this case to 27 Reynolds equations to be considered. The complexity of this physical model underlines the requirement of a fast Reynolds solver. Moreover, the trend throughout the last years to use higher spatial resolutions in order to resolve local phenomena like edge loading in the oil supply further underlines this necessity.

The main task of this work is to develop a fast numerical solver for the discretized Reynolds equation. A well-established method for this purpose is the multigrid method, which can be formulated for linear as well as for nonlinear problems. For a general introduction to multigrid methods, see [4] or [10]. In particular, nonlinear multigrid methods have been

* Received June 2, 2020. Accepted March 3, 2021. Published online on April 27, 2021. Recommended by Marco Donatelli.

$\dagger$ AVL List GmbH, Hans-List-Platz 1,8020 Graz, Austria

(\{michael.reichelt, markus.windisch, guenter.offner\}@avl.com).

${ }^{\ddagger}$ RICAM/ÖAW, Johann Radon Institute, Altenberger Straße 69, 4040 Linz, Austria. 


\section{ETNA}

Kent State University and

Johann Radon Institute (RICAM)

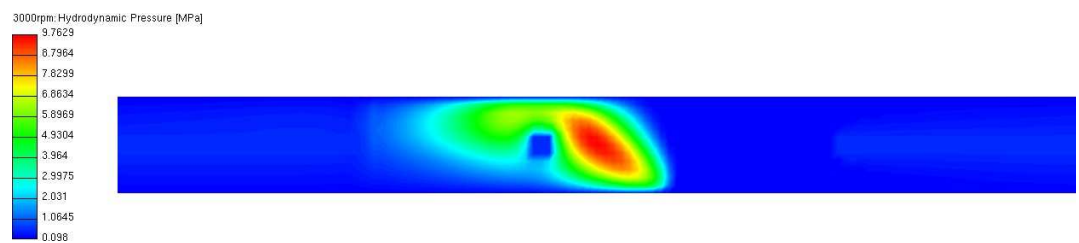

FIG. 1.1. Calculated hydrodynamic pressure distribution for the unrolled main bearing 2 of a 1.9 liter fourcylinder diesel engine with a bore of $80 \mathrm{~mm}$ and a stroke of $92.8 \mathrm{~mm}$ at a crank angle of $280^{\circ}$ (between the firing of the two neighboring cylinders). The engine is running at $3000 \mathrm{rpm}$ at full load.

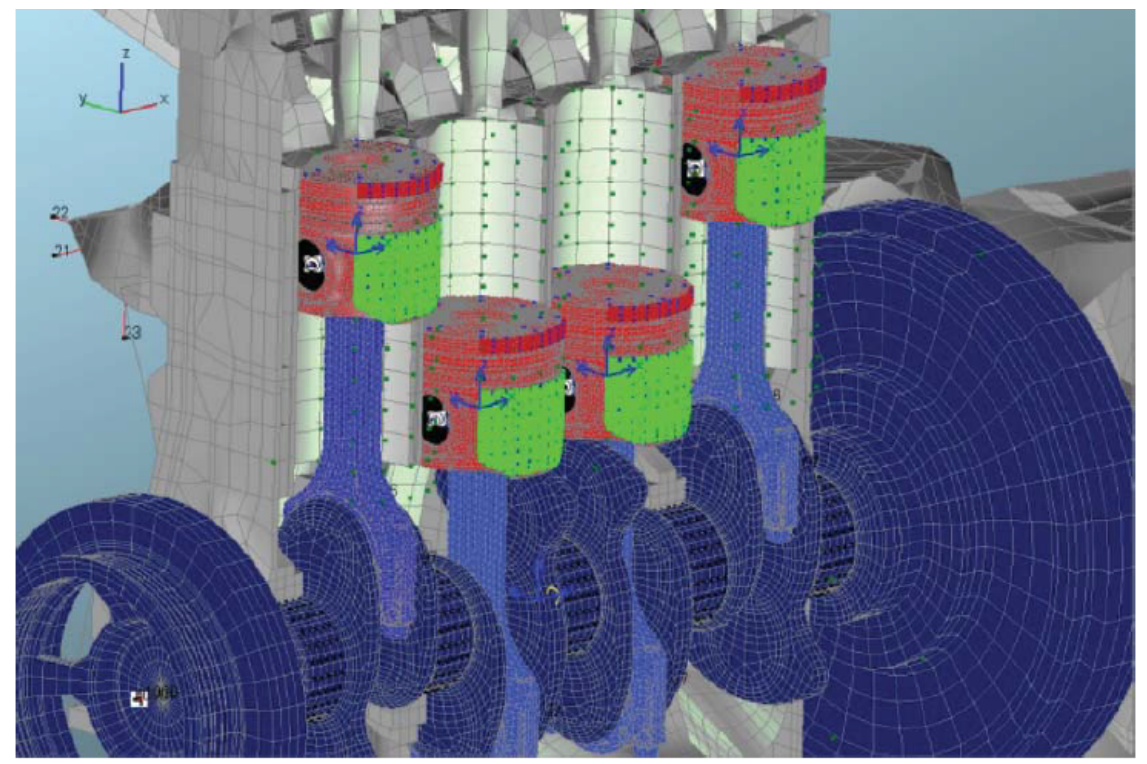

FIG. 1.2. 1.9 liter four-cylinder diesel engine with a bore of $80 \mathrm{~mm}$ and a stroke of $92.8 \mathrm{~mm}$.

investigated for instance by Bobach in the context of flexible bodies and for the Reynolds equation (see [3]). In his work the Reynolds equation and the elastic body deformations are directly coupled. Also, further nonlinearities like variable viscosity and density are considered. The resulting system is therefore highly nonlinear, and a nonlinear multigrid method, like FAS, is appropriate. This is associated with high computational costs since on every grid level a nonlinear problem has to be formulated. Moreover, the necessary steps on each grid level are nonlinear.

General multi-body system solvers are designed more modularly, which allows to solve the Reynolds equation without a direct consideration of elasticity. Furthermore, oil viscosity and density are assumed to be constant during one iteration of the Reynolds solver. Usually, they are determined by a fixed-point iteration.

Hence, the Reynolds equation is linear once the lubrication and the cavitation domains are fixed. This leads to the idea of using a nonlinear solver only on the finest grid and a linear multigrid method to accelerate the global propagation of the solution. The multigrid method used in this work is based on the combination of a geometric and an algebraic approach. The coarse grids and the corresponding restriction and prolongation operators are constructed geometrically. The coarse-grid matrices are constructed in an algebraic way using the Galerkin approach. This approach is highly modular and easy to implement. 
2. The Reynolds equation. The Reynolds equation is a simplification of the NavierStokes equations. It describes the fluid behavior in oil-lubricated contacts. The quantity of interest is the fluid pressure $p$. The equation is given as follows (see [2]):

$$
\underbrace{-\frac{\partial}{\partial x}\left(\alpha^{2} \frac{\partial p}{\partial x}\right)-\frac{\partial}{\partial z}\left(\alpha^{2} \frac{\partial p}{\partial z}\right)}_{\text {Poisseuille term }}+\underbrace{\frac{\partial}{\partial x} \beta}_{\text {Couette term }}+\underbrace{\frac{\partial}{\partial t} \gamma}_{\text {Displacement term }}=0,
$$

where

$$
-\alpha^{2}:=-\frac{\rho h^{3}}{12 \eta}, \quad \beta:=\rho h \frac{\Delta v}{2}, \quad \gamma:=h \rho,
$$

with the fluid density $\rho$, the fluid viscosity $\eta$, the height of the bearing gap $h$, and the relative circumferential sliding velocity of the bearing surfaces $\Delta v$. Without loss of generality, $x$ is the circumferential sliding direction, and $z$ is the axial direction of the bearing. The terms can be interpreted as follows:

- Poisseuille term: Describes the distribution of the pressure without outer influences.

- Couette term: Describes the alteration of the pressure distribution caused by the mass flow due to shear forces.

- Displacement term: Describes the alteration of the pressure distribution due to a change of the clearance height.

2.1. Cavitation. If the pressure in a fluid drops below a certain pressure (the caviation pressure $p_{\text {cav }}$ ), then parts of it will evaporate. Cavitation is the formation of vapor cavities due to this process. One distinguishes between soft (evaporation of gases dissolved in the liquid) and transient (evaporation of the liquid due to critically low pressure) cavitation. The latter is of major interest in engineering because the vapor cavities formed this way implode with increasing pressure. This leads to shock waves, which damage the bearing. The classical Reynolds equation (equation (2.1)) does not describe the phenomenon of cavitation, and it may lead to pressures below the cavitation pressure. A well-established mass conserving approach including cavitation is the JFO model going back to Jakobsson, Floberg and Olson (see [5] and [8]). In this approach it is assumed that the pressure in the cavitation areas is constant $\left(p=p_{\text {cav }}\right)$. Thus, the pressure is given by

$$
p=\max \left\{p_{\text {cav }}, p\right\} .
$$

Under the assumption that the oil density is constant, the occurrence of cavitation means that the gap is not fully filled. This motivates the introduction of the fill ratio

$$
0 \leq \theta \leq 1 \text {, }
$$

which indicates the percentage of the filled volume. This leads to the modified Reynolds equation by substituting $\rho$ by $\theta \rho$ :

$$
\underbrace{-\frac{\partial}{\partial x}\left(\alpha^{2} \frac{\partial p}{\partial x}\right)-\frac{\partial}{\partial z}\left(\alpha^{2} \frac{\partial p}{\partial z}\right)}_{\text {Poisseuille term }}+\underbrace{\frac{\partial}{\partial x}(\theta \beta)}_{\text {Couette term }}+\underbrace{\frac{\partial}{\partial t}(\theta \gamma)}_{\text {Displacement term }}=0,
$$

where the coefficients $\alpha, \beta, \gamma$ are as in equation (2.1). In the lubrication area (no cavitation), $\theta \equiv 1$ holds, and the equation is solved for $p$. In the caviation area, $p \equiv p_{\text {cav }}$ holds, and the equation is solved for $\theta$. This requires knowledge of these areas, which is not existent beforehand. So a solver for this equation has to determine two things: the location of the areas and the pressure or fill ratio, respectively (see [2]). The coefficient $\alpha$ does not need to be adapted since in areas where $\theta<1$ holds, the pressure is constant, and hence its derivative vanishes. 


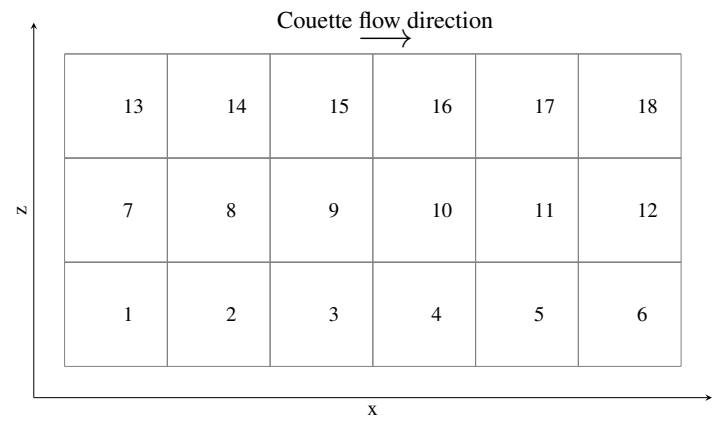

FIG. 2.1. 2D cell grid with cell numbering.

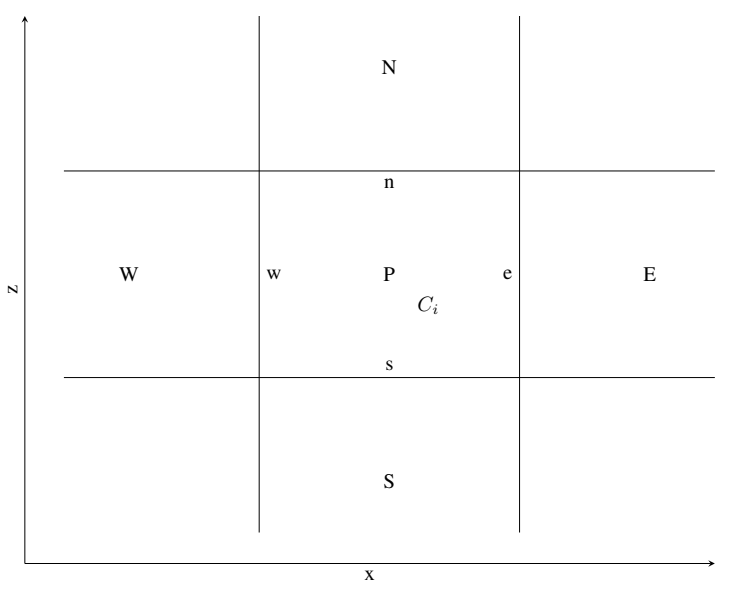

FIG. 2.2. Notation for neighboring cells and edges.

2.2. Discretization of the Reynolds equation. The Reynolds equation is discretized using the finite volume method assuming equidistant divisions in the circumferential and axial directions. For this purpose, the two-dimensional domain is subdivided into rectangular cells of size $\Delta x \times \Delta z$, where $\Delta x$ is the circumferential and $\Delta z$ the axial direction cell size (see Figure 2.1). Without loss of generality, the Couette flow direction is assumed to be in the positive x-direction.

Notation. Originating from the cell of interest $P$, the neighboring cells are denoted by $N, E, S, W$, and the edges of the cell are denoted by $n, e, s, w$ (see Figure 2.2).

For the finite volumes discretization, a mass flow vector is defined as

$$
\dot{m}:=\left(\begin{array}{c}
-\alpha^{2} \frac{\partial p}{\partial x}+\theta \beta \\
-\alpha^{2} \frac{\partial p}{\partial z}
\end{array}\right)
$$

Equation (2.2) is then equivalent to

$$
-\operatorname{div} \dot{m}=\frac{\partial}{\partial t}(\theta \gamma)
$$


Integrating over the area of the $i$ th cell $C_{i}$ leads to

$$
\int_{C_{i}} \frac{\partial}{\partial t}(\theta \gamma) d A=-\int_{C_{i}} \operatorname{div} \dot{m} d A \underbrace{=}_{\text {Gaussian theorem }}-\int_{\partial C_{i}}\langle\dot{m}, n\rangle d s,
$$

where $n$ denotes the normal vector pointing to the exterior of $C_{i}$ and $\langle\cdot, \cdot\rangle$ denotes the standard scalar product. The flow through an edge is approximated to be constant. This leads to

$$
\int_{\partial C_{i}}\langle\dot{m}, n\rangle d s \approx \Delta z\left(\dot{m}_{x}^{e}-\dot{m}_{x}^{w}\right)+\Delta x\left(\dot{m}_{z}^{n}-\dot{m}_{z}^{s}\right) .
$$

The left-hand side of equation (2.3) is approximated by

$$
\int_{C_{i}} \frac{\partial}{\partial t}(\theta \gamma) d A \approx \Delta x \Delta z\left(\frac{\partial}{\partial t}\left(\theta^{P} \gamma^{P}\right)\right) .
$$

This yields

$$
\Delta x \Delta z \underbrace{\left(\frac{\partial}{\partial t}\left(\theta^{P} \gamma^{P}\right)\right)}_{\text {Displacement term }}=\underbrace{\Delta z\left(\dot{m}_{x}^{e}-\dot{m}_{x}^{w}\right)+\Delta x\left(\dot{m}_{z}^{n}-\dot{m}_{z}^{s}\right)}_{\text {Poisseuille and Couette terms }} .
$$

The occurring terms in equation (2.4) are discretized in the following way:

Poisseuille term. The Poisseuille term on an edge of the cell is discretized by geometric averaging in $\alpha$, which has proven in practice to be better than arithmetic averaging. The pressure derivative is discretized using a first-order differential quotient. For the east edge it reads as

$$
\left(-\alpha^{2} \frac{\partial p}{\partial x}\right)^{e} \approx-\alpha^{P} \alpha^{E} \cdot \frac{p^{E}-p^{P}}{\Delta x}
$$

and for the west edge as

$$
\left(-\alpha^{2} \frac{\partial p}{\partial x}\right)^{w} \approx-\alpha^{P} \alpha^{W} \cdot \frac{p^{P}-p^{W}}{\Delta x} .
$$

The north and the south edge terms are discretized analogously.

Couette term. The Couette term on an edge is approximated by an upwind scheme. This means that the quantity of interest on an edge is assumed to have the value of the cell from the Couette flow direction. Since it is here assumed to be from left to right, the value from the left cell is taken. For the east edge, this leads to

$$
(\theta \beta)^{e} \approx \theta^{P} \beta^{P}
$$

and for the west edge to

$$
(\theta \beta)^{w} \approx \theta^{W} \beta^{W} .
$$

Displacement term. The displacement term is approximated by a backward differential quotient, i.e.,

$$
\left(\frac{\partial}{\partial t}(\theta \gamma)\right)(t) \approx \frac{\theta^{P}(t) \gamma^{P}(t)-\theta^{P}(t-\Delta t) \gamma^{P}(t-\Delta t)}{\Delta t},
$$


where $\Delta t$ is the time step size. Hence, the mass flow through the edges read as

$$
\begin{aligned}
& \dot{m}_{z}^{n} \approx \alpha^{P} \alpha^{N} \cdot \frac{p^{P}-p^{N}}{\Delta z}, \\
& \dot{m}_{z}^{s} \approx-\alpha^{P} \alpha^{S} \cdot \frac{p^{P}-p^{S}}{\Delta z}, \\
& \dot{m}_{x}^{e} \approx \alpha^{P} \alpha^{E} \cdot \frac{p^{P}-p^{E}}{\Delta x}+\theta^{P} \beta^{P}, \\
& \dot{m}_{x}^{w} \approx-\alpha^{P} \alpha^{W} \cdot \frac{p^{P}-p^{W}}{\Delta x}+\theta^{W} \beta^{W} .
\end{aligned}
$$

Therefore, the discrete Reynolds equation with the JFO cavitation model for one cell has the following form:

$$
\begin{aligned}
& -\Delta z\left(\alpha^{P} \alpha^{E} \cdot \frac{p^{P}-p^{E}}{\Delta x}+\theta^{P} \beta^{P}+\alpha^{P} \alpha^{W} \cdot \frac{p^{P}-p^{W}}{\Delta x}-\theta^{W} \beta^{W}\right) \\
& -\Delta x\left(\alpha^{P} \alpha^{N} \cdot \frac{p^{P}-p^{N}}{\Delta z}+\alpha^{P} \alpha^{S} \cdot \frac{p^{P}-p^{S}}{\Delta z}\right) \\
& +\Delta x \Delta z \cdot \frac{\theta^{P}(t) \gamma^{P}(t)-\theta^{P}(t-\Delta t) \gamma^{P}(t-\Delta t)}{\Delta t}=0 .
\end{aligned}
$$

In the lubrication area, $\theta \equiv 1$, and equation (2.5) has to be solved for $p$. In the cavitation area it has to be solved for $\theta$, and the pressure is set to $p \equiv p_{\text {cav }}$. For the purpose of notational simplicity, a cell-type vector $\xi$ is introduced, which contains the information whether a cell is currently defined as a pressure (lubrication) or a cavitation cell. Consequently, it has one of the following states

$$
\xi_{i}=\left\{\begin{array}{l}
\text { pressure, } \\
\text { cavitation, }
\end{array}\right.
$$

corresponding to the state of the $i$ th cell. For a fixed $\xi$, the system of equations resulting from (2.5) can be written as a linear system

$$
A(\xi) x(\xi)=f(\xi),
$$

where $x_{i}$ contains either the pressure or the fill ratio of the $i$ th cell (depending on $\xi$ ).

Boundary Conditions. Boundary conditions are realized by setting cells on the grid to a prescribed pressure. Imposing boundary conditions at the edges of the bearings is required, but additional boundary conditions can be set inside of them. This is needed for instance to represent oil supply drillings.

2.3. Solution techniques. Since the discretized Reynolds equation (equation (2.6)) is of matrix type for fixed domains, linear solution schemes combined with an adaption of the lubrication and cavitation domains during the iterations are promising. A simple approach is to start with an initial $\xi_{0}$, solve the associated linear system, alter the cell types according to the solution, and repeat until convergence is reached. The major disadvantage of this method is that it requires the solution of a linear system in every step. Furthermore, numerical experiments have shown that it converges rather slowly. This is due to the fact that the influence of the pressure cells on the cavitation area is very local. Therefore, cell switches from cavitation to pressure only happen in the area very close to the boundary even if the 
surrounding pressure is high. Hence the domain adaption driven by the global solution is dilatory. A method that utilizes this local character is the Reynolds-Gauss-Seidel iteration. The classical Gauss-Seidel method is used for solving linear systems. The algorithm loops through the matrix rows and solves for the variable matching the diagonal entry using the already calculated values of the intermediate solution. For a general introduction into this topic, see, for instance, [9]. The Gauss-Seidel method can be modified in order to solve the nonlinear system of equations that arises from the discretization of the Reynolds equation. If during the row iteration the cell type corresponding to the current row changes (i.e., the pressure falls below $p_{\text {cav }}$ or the fill ratio above 1), then the entries of the system matrix associated with this cell are recalculated. Based on the new matrix entries, the cell value is recomputed. Relaxation methods help to improve convergence. For a more detailed description, see [6]. Parallelization techniques like red-black labelling are also possible. However, this method suffers, like linear relaxation methods, from decreasing efficiency with increasing grid resolution. A combination of successively solving linear systems and the Reynolds-Gauss-Seidel method uses both the global information propagation by directly solving the system and the rapid domain adaption from the Gauss-Seidel-like iteration.

Algorithm 1 (Combined Algorithm). Let $A(\xi) x(\xi)=f(\xi)$ be the discretized Reynolds equation (equation (2.6)) depending on $\xi$ and $A(\xi) \in \mathbb{R}^{N \times N}$. Furthermore, let $R G S(x, \xi, \omega, k)$ be the function that applies $k$ Reynolds-Gauss-Seidel iterations with relaxation parameter $\omega$ (see [6]). Then the combined algorithm is given by

while domains change do

$$
x=(A(\xi))^{-1} f(\xi)
$$

adapt $\xi$ with the information from $x$

$(x, \xi)=R G S(x, \xi, \omega, k)$

\section{end while}

Here $k$ is usually chosen in the range of 2 to 5 .

This algorithm converges rapidly, but the computational effort of the direct inversion of $A$ is high, especially for large systems, and of order $\mathcal{O}\left(N^{3}\right)$. The idea is to replace the matrix inversion by a multigrid method which has optimal efficiency $(\mathcal{O}(N \log N))$.

3. The multigrid method. In this section a multigrid method for the solution of the nonlinear system of equations associated with equation (2.6) is developed. The general linear multigrid cycle, the so-called $\mu$-cycle, is used. For a general introduction into this topic, see [4] or [10]. For notational purposes the grid system is formally defined as follows:

DEFINITION 3.1 (Grid System). Let $A x=b$ be the system to solve on a cell system $C_{0}$. Further, let there be given $n$ coarser grids $C_{1}, \ldots, C_{n}$, where $C_{l}$ is coarser than $C_{l-1}$, prolongation operators $P_{1}, \ldots, P_{n}$, and restriction operators $R_{1}, \ldots, R_{n}$, where

$$
P_{l}: C_{l} \rightarrow C_{l-1}
$$

prolongates a solution from the lth to the $(l-1)$ st grid and

$$
R_{l}: C_{l-1} \rightarrow C_{l}
$$

restricts from the $(l-1)$ st to the lth grid. Further, let $A_{0}, \ldots, A_{n}$ be the system matrices on each grid level. The grid system $G$ is defined as

$$
G:=\left(G_{1}, G_{2}, \ldots, G_{n}\right),
$$

where

$$
G_{l}:=\left(A_{l}, P_{l}, R_{l}\right) .
$$


Then the $\mu$-cycle can be defined as in Algorithm 2.

ALGORITHM 2 ( $\mu$-cycle). Let $A x=b$ be a linear system. Furthermore, let $G$ be a grid system as in Definition 3.1 and $S\left(v^{l}, A_{l}, f^{l}\right)$ a smoother (e.g., a Gauss-Seidel iteration). Let $A_{0}=A, f^{0}=b$. Denote the approximate solution on the lth level by $v^{l}$. Then, the $\mu$-cycle is given by

$$
\begin{aligned}
& v^{l} \leftarrow M_{\mu}\left(v^{l}, f^{l}, \nu_{1}, \nu_{2}\right) \\
& \text { if } \Omega^{l} \text { is coarsest grid then } \\
& \quad v^{l}=A_{l}^{-1} f^{l} \\
& \text { else } \\
& \quad v^{l}=S\left(v^{l}, A_{l}, f^{l}\right) \nu_{1} \text {-times } \\
& \quad f^{l+1}=R_{l+1}\left(f^{l}-A_{l} v^{l}\right) \text { restrict residual to coarser level } \\
& \quad v^{l+1}=0 \text { set the initial guess for the coarser level } \\
& \quad v^{l+1}=M_{\mu}\left(v^{l+1}, f^{l+1}, \nu_{1}, \nu_{2}\right) \mu \text {-times } \\
& \quad v^{l}=v^{l}+P_{l+1} v^{l+1} \text { correct solution on current level with coarse-grid information } \\
& v^{l}=S\left(v^{l}, A_{l}, f^{l}\right) \nu_{2} \text {-times } \\
& \text { end if }
\end{aligned}
$$

The algorithm does not define how the prolongation, restriction, and system matrices should be constructed. The efficiency of the algorithm depends heavily on these choices, and there are various options. Hence, it is necessary to adapt them to the given problem.

3.1. Prolongation, restriction, and coarse-grid matrix. This section covers the prolongation, the restriction, and the construction of the coarse-grid systems. Only 2D restriction and prolongation operators are defined. A frequently-used approach is to construct a prolongation operator $P$ and then choose the restriction operator as $R=c P^{\top}$, where $c$ is a constant.

3.1.1. Interpolation for cell-systems. Let $\Omega \subset \mathbb{R}^{2}$ be a domain, which is partitioned into a set $C_{h}=\left\{c_{h}^{1}, c_{h}^{2}, \ldots\right\}$ of finer cells with $n_{h}$ elements and a set $C_{H}=\left\{c_{H}^{1}, c_{H}^{2}, \ldots\right\}$ of coarser cells with $n_{H}$ elements, i.e.,

$$
\bigcup_{c_{h}^{i} \in C_{h}} c_{h}^{i}=\bigcup_{c_{H}^{i} \in C_{H}} c_{H}^{i}=\bar{\Omega} .
$$

Furthermore, the elements of both sets shall be disjoint, i.e.,

$$
\left(c_{H}^{i}\right)^{\circ} \cap\left(c_{H}^{j}\right)^{\circ}=\emptyset=\left(c_{h}^{i}\right)^{\circ} \cap\left(c_{h}^{j}\right)^{\circ} \quad \forall i \neq j .
$$

In addition, every cell in $C_{H}$ (parent cell) must consist of cells from $C_{h}$ (children cells), i.e.,

$$
\forall c_{H} \in C_{H}: c_{H}=\bigcup_{C_{h} \ni c_{h}^{i} \subset c_{H}} c_{h}^{i} .
$$

Let $f_{H} \in\left\{g_{H} \mid g_{H}: C_{H} \rightarrow \mathbb{R}\right\}$ be a function represented as a vector (which is in $\mathbb{R}^{n_{H}}$; the $i$ th component of the vector corresponds to the value of $f_{H}\left(c_{H}^{i}\right)$ ). Then, the prolongation operator $P$ assigns to every child cell the function value of its parent cell. This can be written in matrix form as

$$
P \in \mathbb{R}^{n_{h} \times n_{H}}, \quad P[i, j]= \begin{cases}1 & \text { if } c_{h}^{i} \subseteq c_{H}^{j}, \\ 0 & \text { if } c_{h}^{i} \nsubseteq c_{H}^{j} .\end{cases}
$$

The restriction operator $R$ can be applied to a vector $f_{h} \in \mathbb{R}^{n_{h}}$, which again represents a function on the finer cell system, and is chosen to assign to every parent cell the averaged 
value of its children cells. In matrix form it can be written as

$$
R \in \mathbb{R}^{n_{H} \times n_{h}}, \quad R[i, j]= \begin{cases}\frac{1}{k(i)} & \text { if } c_{h}^{j} \subseteq c_{H}^{i}, \\ 0 & \text { if } c_{h}^{j} \nsubseteq c_{H}^{i},\end{cases}
$$

where $k(i)$ denotes the number of child cells of $c_{H}^{i}$.

3.1.2. Construction of coarse-grid matrices. Based on the restriction and prolongation operators, the coarse-grid matrix is constructed with the prolongation and restriction matrices. Let the fine-grid system be denoted by $A_{h} x_{h}=b_{h}$, and let $\tilde{x}_{h}$ be the approximate solution after the application of a smoother. Then the residual is given as

$$
r_{h}=A_{h} \tilde{x_{h}}-b_{h}=A_{h}\left(\tilde{x}_{h}-x_{h}\right)=A_{h} e_{h},
$$

where $e_{h}$ denotes the error on the fine grid. Let $R$ be the restriction from the fine grid to the coarse grid and $P$ the prolongation from the coarse grid to the fine grid. Since the highly oscillating parts of $e_{h}$ have been removed by applying a smoother, it can be approximated by the prolongation of the coarse-grid error

$$
e_{h} \approx P e_{H}
$$

where $e_{H}$ denotes the error on the coarse grid. Then, for equation (3.1) it holds that

$$
r_{h}=A_{h} e_{h} \approx A_{h} P e_{H} .
$$

Applying $R$ to equation (3.2) leads to

$$
r_{H}:=R r_{h}=R A_{h} P e_{H}=: A_{H} e_{H}
$$

In general the coarse-grid system matrix is often defined as

$$
A_{H}=c R A_{h} P
$$

where $c$ is a constant. $R A_{h} P$ is called the "Galerkin-Operator". Here, the Galerkin operator is multiplied by a constant for a better approximation of the discretization matrix, which is directly constructed from the coarse grid.

3.2. Specific coarse-grid construction. The generation of the coarser grids is done by merging, starting in the lower left corner of the cell grid. If possible, four cells are merged together. If necessary, two cells are merged together or one cell is directly ported to the next grid level. Figure 3.1 illustrates this scheme. The coarse-grid cell is the parent cell of the fine-grid cells with the same index.

A suitable coarse-grid matrix can be defined by

$$
A_{H}=\frac{1}{2} R A_{h} P
$$

The factor $\frac{1}{2}$ is motivated by the discretization of the Laplace equation. When discretizing the Laplace equation using the finite volume method directly on the coarse and the fine grid, the corresponding matrices fulfill equation (3.3) as one easily calculates (see [4]). Since the Reynolds and the Laplace equation are very similar, it is also a good approximation for the Reynolds coarse-grid matrix. 


\begin{tabular}{|r|r|r|r|r|r|r|r|r|r|r|}
\hline 13 & 13 & 14 & 14 & 15 & 15 & 16 & 16 & 17 & 17 & 18 \\
\hline 13 & 13 & 14 & 14 & 15 & 15 & 16 & 16 & 17 & 17 & 18 \\
\hline 7 & 7 & 8 & 8 & 9 & 9 & 10 & 10 & 11 & 11 & 12 \\
\hline 7 & 7 & 8 & 8 & 9 & 9 & 10 & 10 & 11 & 11 & 12 \\
\hline 1 & 1 & 2 & 2 & 3 & 3 & 4 & 4 & 5 & 5 & 6 \\
\hline 1 & 1 & 2 & 2 & 3 & 3 & 4 & 4 & 5 & 5 & 6 \\
\hline
\end{tabular}

\begin{tabular}{|c|c|c|c|c|c|}
\hline 13 & 14 & 15 & 16 & 17 & 18 \\
\hline 7 & 8 & 9 & 10 & 11 & 12 \\
\hline 1 & 2 & 3 & 4 & 5 & 6 \\
\hline
\end{tabular}

FIG. 3.1. Merging fine grid cells.

3.3. Reynolds-Gauss-Seidel iteration with multigrid. Since the multigrid method cannot be easily adapted to perform the cell alteration between cavitation and pressure cells, it is advantageous to use it between the applications of the Reynolds-Gauss-Seidel algorithm (described in [6]) as some sort of preconditioner. In contrast to the usual geometrical multigrid approach, the proposed method constructs its hierarchy through the Galerkin operator instead of rediscretizing the equation on the coarser grids. In this way the virtues of both approaches are used since the algebraic coarse-grid construction is fast once prolongation and restriction operators are defined. On the other hand, the usually more costly algebraic construction of these operators is avoided.

ALGORITHM 3 (Reynolds-Gauss-Seidel iteration with multigrid.). Let $A(\xi) x(\xi)=f(\xi)$ be the discretized Reynolds equation (2.6) depending on $\xi$. Then the Reynolds-Gauss-Seidel iteration with multigrid is given by

choose $\xi$

choose $x(\xi)$

while $\|A(\xi) x(\xi)-f(\xi)\|>$ tolerance do

$x(\xi)=M_{\mu}\left(x(\xi), f(\xi), \nu_{1}, \nu_{2}\right)$ apply multigrid $n$-times

adapt $\xi$ with the information from $x$

set the values in $x$ whose cell types changed to the

equivalent pressure/cavitation

apply Reynolds-Gauss-Seidel algorithm $m$-times

\section{end while}

With this modifications, the Reynolds-Gauss-Seidel algorithm drastically accelerates the determination of the lubrication and cavitation areas. One could think about turning it off after the cell types are fixed, but numerical experiments have shown that it stabilizes and accelerates the convergence of the procedure for a relatively low computational cost.

The parameters of this algorithm are listed in Table 3.1. Depending on the smoother, the parameters $\nu_{1}$ and $\nu_{2}$ have to be chosen sufficiently large to maintain decent smoothing 
properties, but also not too large since after a certain amount of smoothing steps, the error will not become significantly smoother, which only leads to higher computational costs without benefit. In most cases, the value of $n=1$ is the best choice because when the lubrication and cavitation areas are not stable, a better solution of the linear system is useless since the system changes in the next step.

TABLE 3.1

Parameters for the Reynolds-Gauss-Seidel iteration with multigrid (Algorithm 3).

\begin{tabular}{ccc} 
parameter & reasonable values & comment \\
\hline$\mu$ & 1,2 & V- or W-cycle \\
$\nu_{1}$ & $1-5$ & \# Smoothing steps before restriction \\
$\nu_{2}$ & $1-5$ & \# Smoothing steps after prolongation \\
$n$ & $1-2$ & \# multigrid cycles in each iteration \\
$m$ & $1-4$ & \# Reyn.-GS steps in each iteration
\end{tabular}

REMARK 3.2. The robustness and efficiency of the Reynolds-Gauss-Seidel iteration with multigrid algorithm can be significantly improved by reformulating the discretized Reynolds equation to an "equivalent pressure" formulation. In this formulation a "negative pressure" is calculated for the cavitation cells of such amount that would be needed to fill the cell up completely. Therefore, the diagonal of the matrix is invariant under cell changes, and the diagonal values are usually of the same magnitude, which is beneficial to the multigrid method. The "negative pressure" can be transformed to a fill ratio after the multigrid process. This approach is used in this work.

4. Results. The Reynolds-Gauss-Seidel-multigrid algorithm (see Algorithm 3) is implemented in a multi-body solver framework (AVL EXCITE ${ }^{\mathrm{TM}}$ [1]). The framework uses backward differentiation formulas with adaptive time step size control (see [7]) in the time domain. Each time step iterates over the body equations of motion and contact equations, which are represented by a Reynolds equation in case of each particular oil-lubricated contact.

4.1. Physical model of a single radial slider bearing. The model, which is used for demonstration, consists of a bearing wall, a bearing pin, and the oil layer between them. It represents the main bearing 2 of the engine depicted in Figure 1.2. A topological representation is given in Figure 4.1.

4.2. Convergence of the Reynolds-Gauss-Seidel iteration with multigrid algorithm. Well-implemented multigrid methods for linear equation systems show an exponential convergence (respectively, linear convergence in a logarithmic plot) independently of the system size. A similar behavior is observed using the Reynolds-Gauss-Seidel iteration with multigrid algorithm. In the phase when the cell types are not fixed (cycle 1 to 7), the convergence trend is perturbed, but even in this region it is almost linear in the logarithmic plot, however with a smaller gradient. This is illustrated in Figure 4.2(a). The initial guess is taken as all cells being pressure cells with cavitation pressure. One observes that in the phase where the cell types are massively changing, the convergence trend is nearly independent of the multigrid parameters. However, after this phase, additional smoothing steps and the $\mathrm{W}$-cycle $(\mu=2)$ pay off. This is illustrated in Figure 4.2(b), where only that part of the solution process is displayed where the cell types are fixed. The used smoother is Gauss-Seidel-Red-Black; see [10]. Starting with cycle 10, the relative residual stays approximately the same, which is most likely due to floating-point accuracy. In practice, algorithms with fewer smoothing iterations can be faster. Further, the initial guess is usually much better since it can be taken from the last time step, 


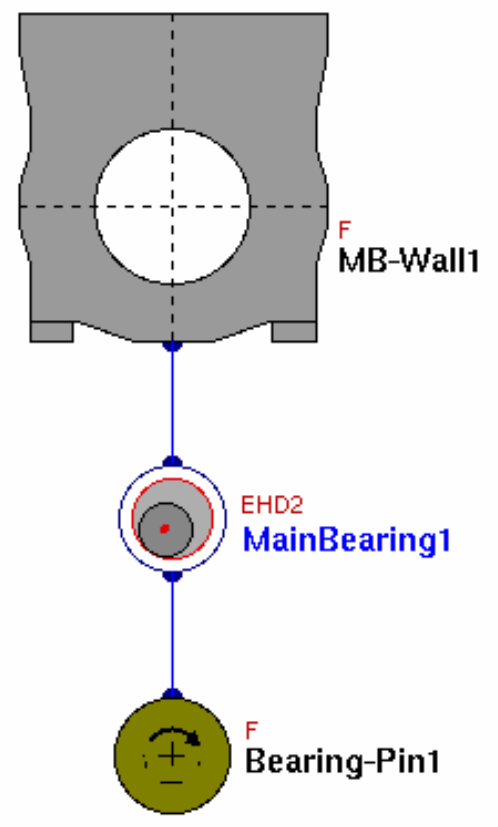

FIG. 4.1. Topological representation of the physical model.

which reduces the number of cycles needed for the domain determination. For the illustrations, one Reynolds iteration of the multi-body solver is taken, the initial values are set accordingly, and three gridlevels are used. A typical result of such a calculation is illustrated in Figure 1.1.

4.3. Comparison of the simulation times. The Reynolds-Gauss-Seidel method without multigrid is compared to the Reynolds-Gauss-Seidel method with multigrid in terms of simulation time. Here, $720^{\circ}$ are simulated for different discretizations and journal speeds. Furthermore, the mere solver time is also recorded. The stopping criterion for the algorithm using multigrid is when the relative residual falls below $10^{-5}$, and the remaining parameters of the Reynolds-Gauss-Seidel iteration with multigrid are as in Table $3.1 \mu=1, \nu_{1}=2, \nu_{2}=3$, $n=1, m=3$. The used smoother is Gauss-Seidel-Red-Black; see [10]. Due to historical reasons of the multi-body framework, the convergence criteria for the Reynolds-Gauss-Seidel iteration without multigrid are more complicated. The solver stops when the relative difference between two iterations falls below a certain level. Furthermore, the number of iterations is bounded by a lower and an upper limit. Since the simulation fails for higher grid resolutions, if the parameters are chosen too weak, the first set of parameters that does not lead to simulation crashes is chosen:
Relative Difference
$0.001 \%$
Maximal Iterations
Minimal Iterations
4.

With these settings, the Reynolds-Gauss-Seidel method without multigrid also reaches a relative residual of $10^{-5}$ in most solution procedures. It must furthermore be noted that even if the convergence criterion of the Reynolds-Gauss-Seidel iteration with multigrid is set to $10^{-5}$, it usually achieves a significantly smaller residual. With these convergence criteria, the multigrid version outperforms the other solver. Performance results obtained for different 


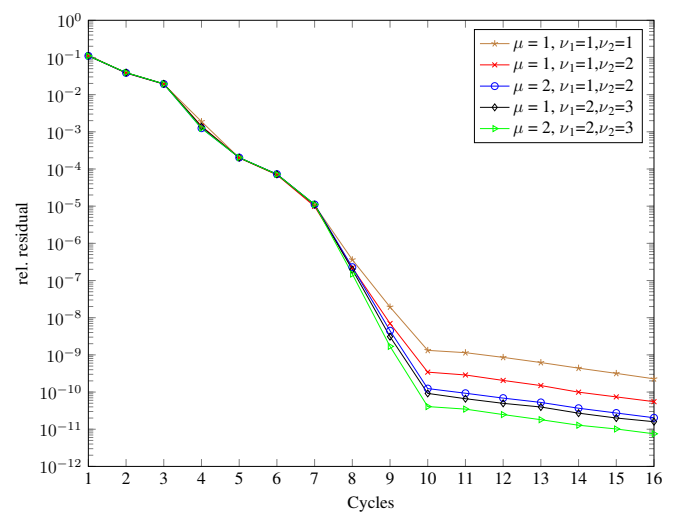

(a) Overall solution process.

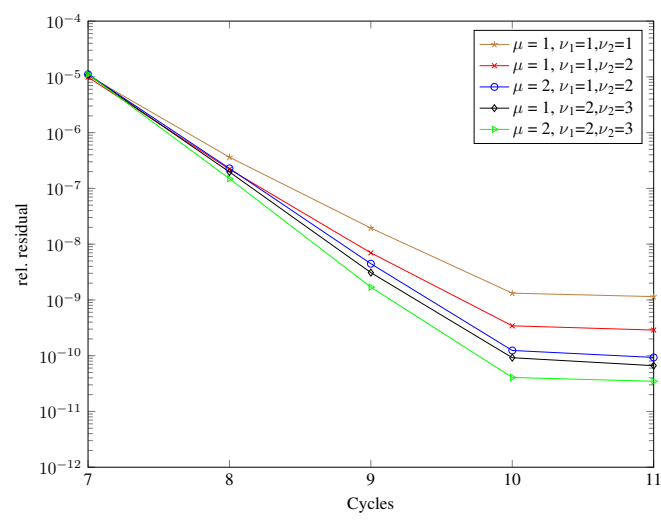

(b) Fixed domains.

FIG. 4.2. Convergence trend of the Reynolds-Gauss-Seidel iteration with multigrid algorithm for different parameters $(n=1, m=3), 3$ gridlevels, discretization: $1024 \times 128$.

engine running conditions $(500,3000$, and $5000 \mathrm{rpm})$ are displayed in Figure 4.3-4.5. It is visible that the percentage of the simulation time taken by the solver is increasing drastically with the grid resolution of the non-multigrid solver, while the solver using multigrid takes up an almost constant percentage for higher resolutions. The resolution in the diagrams is given by the number of circumferential cells times the number of axial cells.

5. Conclusion. It has been shown that multigrid methods can drastically reduce the simulation time and increase the robustness for the simulation of radial slider bearings with high resolutions, even though the same equation system as for the Gauss-Seidel method is solved hence yielding the same results. This facilitates the possibility to get a more detailed view on the pressure distribution at a reasonable amount of simulation time. The presented approach is generic so that it is also possible to implement this method for other elastohydrodynamic contacts like axial slider bearings or hydrodynamic piston-liner contacts.

Acknowledgments. This work was done in collaboration with AVL List who has also provided technical support. Furthermore, part of this work has been supported in the framework of "Industrielle Kompetenzzentren" by the Austrian Ministerium für Wirtschaft, Jugend und Familie and by the government of Upper Austria. 
ETNA

Kent State University and

Johann Radon Institute (RICAM)

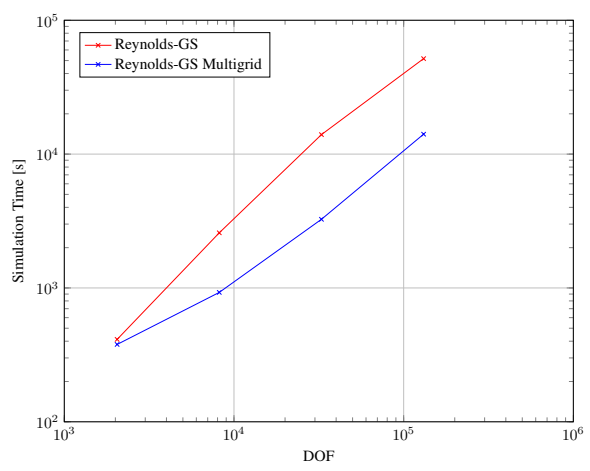

(a) Simulation time.

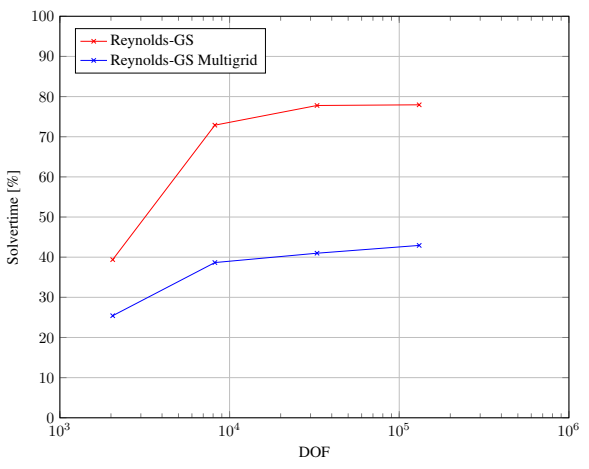

(b) Percentage of simulation time taken by the solver.

FIG. 4.3. Engine running at $500 \mathrm{rpm}, 720^{\circ}$ crank angle simulated, discretizations: $128 \times 16,256 \times 32,512 x 64$, $1024 \times 128$ (circumferential $\times$ axial cells).

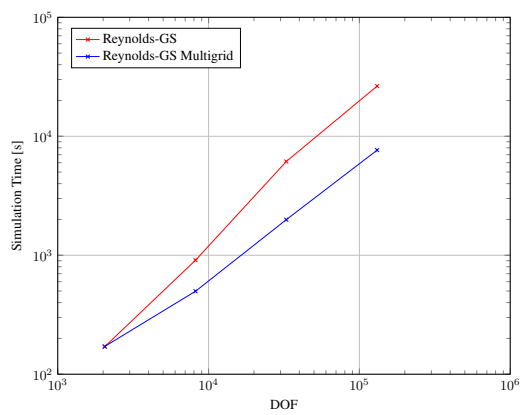

(a) Simulation time.

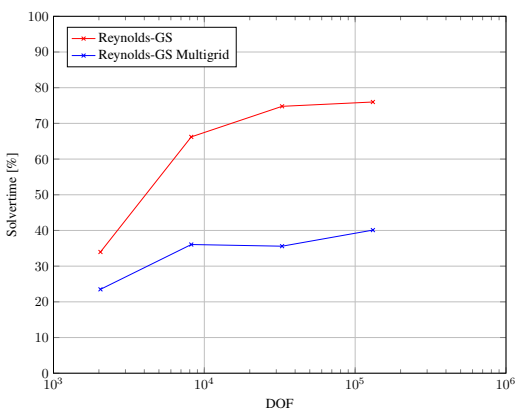

(b) Percentage of simulation time taken by the solver.

FIG. 4.4. Engine running at $3000 \mathrm{rpm}, 720^{\circ}$ crank angle simulated, discretizations: $128 \times 16,256 \times 32,512 \times 64$, $1024 \times 128$ (circumferential $\times$ axial cells $)$.

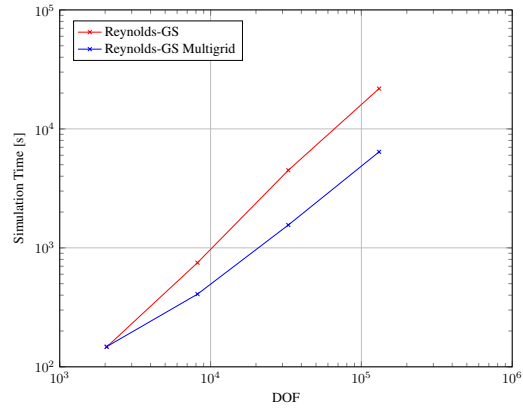

(a) Simulation time.

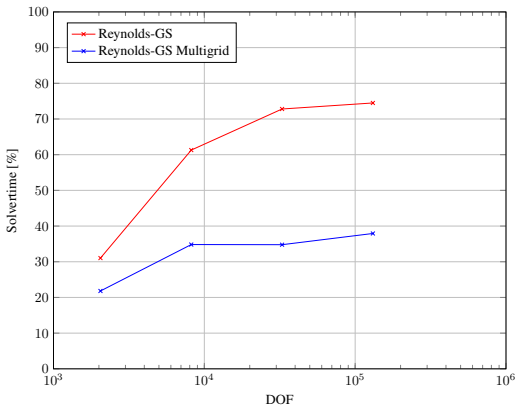

(b) Percentage of simulation time taken by the solver.

FIG. 4.5. Engine running at $5000 \mathrm{rpm}, 720^{\circ}$ crank angle simulated, discretizations: $128 \times 16,256 \times 32,512 \times 64$, $1024 \times 128$ (circumferential $\times$ axial cells). 


\section{ETNA}

Kent State University and

Johann Radon Institute (RICAM)

\section{REFERENCES}

[1] AVL List GmbH., AVL-EXCITE, Theory, AVL List GmbH., Graz, 2017.

[2] D. BARTEL, Simulation von Tribosystemen: Grundlagen und Anwendungen, Vieweg+Teubner, Wiesbaden, 2010.

[3] L. ВовасH, Simulation dynamisch belasteter Radialgleitlager unter Mischreibungsbedingungen, Shaker, Düren, 2008.

[4] W. L. BRIGGS, A Multigrid Tutorial, SIAM, Philadelphia, 1987.

[5] B. Jakobsson And L. Floberg, The Finite Journal Bearing, Considering Vaporization, Chalmers Tekniska Högskolas Handlingar, Gothenburg, 1957.

[6] J. KRASSER, Thermo-Elastohydrodynamic Analysis of Dynamically Loaded Radial Slider Bearings, PhD. Thesis, TU Graz, Graz, 1996.

[7] G. OFFNER, Friction power loss simulation of internal combustion engines considering mixed lubricated radial slider, axial slider and piston to liner contacts, Tribology Trans., 56 (2013), pp. 503-515.

[8] K. Olsson, Cavitation in Dynamically Loaded Bearings, Chalmers Tekniska Högskolas Handlingar, Gothenburg, 1965.

[9] J. StOeR AND R. BuliRsCh, Numerische Mathematik 2, Springer, Berlin, 2005.

[10] U. Trottenberg, C. Oosterlee, And A. Schuller, Multigrid, Academic Press, San Diego, 2001. 